\title{
TISSUE MICROENVIRONMENTS WITHIN THE NASAL MUCOSA OF RAINBOW TROUT COMPARTIMENTALIZE NASAL IMMUNE RESPONSES
}

\author{
Sepahi $A^{1}$, Casadei $E^{1}$, Tacchi $\mathbf{L}^{1}$, LaPatra $\mathrm{SE}^{2}$, Salinas $\mathbf{I}^{*}$ \\ ${ }^{1}$ Center of Evolutionary and Theoretical Immunology Department of Biology University of New Mexico, \\ New Mexico, USA. \\ ${ }^{2}$ Research Division Clear Springs Foods Inc, Buhl, Idaho, USA.
}

\begin{abstract}
Mucosal surfaces are multifunctional barriers that need to orchestrate a variety of physiological functions. One of the mechanisms by which mucosal surfaces carry immunological functions without compromising other physiological roles is the establishment of regional immunity. Regional immunity can be generated by selective expression of chemokines, chemokine receptors and adhesion molecules within mucosal microenvironments. In the olfactory organ of animals, immune responses may result in damage to the olfactory sensory neurons and, as a consequence, compromise olfaction. The olfactory organ of teleost fish is histologically divided into two distinct areas, a sensory or neuroepithelial region in the lateral regions and valleys if the lamellae and a non-sensory region located at the tip of each lamella characterized by presence of goblet cells. Thus, we hypothesized the presence of two unique immune microenvironments corresponding with these two regions in rainbow trout. In line with our hypothesis, we found clusters of $\mathrm{CD}^{+} \mathrm{T}$ cells at the mucosal regions of lamellae whereas few numbers were found in the neuroepithelium. Nasal CD8 ${ }^{+} \mathrm{T}$ cells displayed a mucosal phenotype similar to those found in the gut. Laser capture microdissected tip versus lateral samples of control trout olfactory tissue were collected and RT-qPCR revealed a higher expression of the chemokine and chemokine receptor CCL19 and CCR7, the adhesion molecules L-selectin, ICAM, VCAM, and integrin $\beta 2$ and several T cell markers in the mucosal tip compared to the neuroepithelium. Moreover, MHC-II immunostaining indicated that the mucosal tip preferentially participates in antigen presentation within the trout nasal mucosa. Finally, in response to nasal vaccination, cell proliferation occurs in the tip mucosal region and not the neuroepithelium. Our results support the idea of compartmentalized immune responses within the nasal mucosa of teleosts that likely evolved as a sparing mechanism to protect olfactory sensory function.
\end{abstract}

\section{KEYWORDS}

Regional immunity, NALT, trout, nasal immunity, mucosal immunity

*Corresponding author. Tel.: +1 505-277-0039

E-mail address: isalinas@unm.edu 